\title{
Comportement mécanique d'un sable homométrique stabilisé
}

\author{
Mechanical behaviour of fine stabilised sand
}

\author{
J. BERTOZZI \\ Laboratoire de Construction Civile et Maritime*
}

Rev. Franç. Géotech. n 60 , pp, 51-59 (juillet 1992)

\section{Résumé}

Afin d'étudier le comportement mécanique d'un sable homométrique stabilisé par le liant "cendres volantes-chaux», nous avons suivi l'évolution de la courbe intrinsèque de ce matériau en fonction des trois paramètres : temps, pourcentage de cendres volantes, pourcentage de chaux.

Les résultats des essais de compression triaxiale montrent que la courbe intrinsèque, assimilée à une droite, évolue suivant un parallélisme en fonction du temps, quel que soit le pourcentage de cendres volantes et de chaux.

On propose :

- d'une part, une loi de variation de $R_{c}\left(R_{c}=f\left(R_{c \alpha}\right)\right)$ qui permet, à partir d'un essai de compression simple effectué à 7 jours, de prédire la résistance du sable stabilisé à un an, et par suite, à un âge quelconque compris entre 7 et 360 jours ;

- d'autre part, un abaque qui, à partir de la résistance à la compression simple du sable pur compacté à l'OPM, définit la composition du mélange qui atteindrait les caractéristiques désirées à un âge donné.

\section{Abstract}

In order to study the mechanical behaviour of fine sand (same size sand) stabilised with a fly-ash and lime mixture, we have followed the evolution of the intrinsic curve of this mixture as a function of age, the percentage of fly-ash, and the percentage of lime.

The results of triaxial compression tests show that the evolution of the intrinsic curve, which is assimilated to a straight line, evolves in a regular upward parallel movement with time, wathever the percentage of fly-ash, and lime are.

From these results, we can propose :

- on one hand a variation law for the simple compression test $\left(R_{c}=f\left(R_{c \alpha}\right)\right)$ which allows us to predict the resistance at the end of one year, from a simple compression test done on the seventh day and, as a result, to any age between 7 and 360 days;

- on an other hand, one abacus from the value of the simple compression resistance of pure sand compacted with OPM (Optimum Modified Proctor) characteristics, defines the composition of the mixture which attains the required characteristics at any age. 


\section{INTRODUCTION}

Depuis quelques dizaines d'années, l'augmentation rapide de la consommation en sable, dans le domaine routier et celui de la construction immobilière, est telle que de sérieux problèmes d'approvisionnement se posent actuellement. Ces problèmes sont d'autant plus cruciaux que les sables fins (de moins bonne qualité et des plus répandus tant en France que dans les autres pays du monde), ont été délaissés au profit des sables nobles (sables alluvionnaires).

Les gisements non inextinguibles de ces derniers, les atteintes portées à l'environnement, les nuisances, les affouillements (ex ; pont de Wilson, sur la Loire, à Tours) dus à leur exploitation, ont conduit à une réglementation plus stricte de leur extraction. De plus, les sables, comme tous les granulats, sont des matériaux pondéreux dont le coût de transport devient de plus en plus élevé vis-à-vis du coût de production.

Pour toutes ces raisons, il serait économiquement intéressant pour plusieurs régions, de substituer, en totalité ou en partie, aux granulats habituellement utilisés, des sables fins (sables d'origine marine, sables éoliens). Or ces sables fins, de par leur granulométrie souvent homométrique et leur forme, possèdent des qualités médiocres. Aussi est-il indispensable de les amender ou de les stabiliser pour une utilisation plus rationnelle.

Ainsi, dans l'espoir d'élargir le champ d'exploitation des sables fins très répandus en France, nous avons étudié la stabilisation d'un sable de dune par le liant hydraulique: cendres volantes-chaux [4].

Cette étude s'est faite par le suivi de l'évolution de la courbe intrinsèque, assimilée à une droite. Les résultats d'essais de compression triaxiale, effectués sur des éprouvettes compactées à l'Optimum Proctor Modifié nous ont permis de définir les lois de comportement des caractéristiques mécaniques, angle de frottement interne $\Phi$ et cohésion c, en fonction de trois variables : temps, pourcentage de cendres volantes, pourcentage de chaux, et d'en déduire l'évolution de la résistance à la compression simple ainsi que celle du rapport $R_{t} / R_{c}$.

D'autre part, pour exploiter ces résultats obtenus à partir d'essais triaxiaux, pour des applications pratiques simples, nous avons défini la loi de variation de $R_{c}$ $=f\left(R_{c \propto}\right)$, ainsi qu'un abaque permettant de déterminer le traitement de stabilisation à effectuer sur le sable, connaissant la résistance à la compression simple $\mathrm{R}_{c o}$ du sable pur.

\section{MATÉRIAU}

Le matériau étudié est un sable de dune (fig. 1) stabilisé au liant hydraulique.

L'allure de la courbe granulométrique révèle une pente relativement forte qui caractérise un matériau bien trié, mal gradué, qui est dit « homométrique » : plus de $75 \%$ d'éléments supérieurs à $80 \mu \mathrm{m}$ sont inférieurs à $0,5 \mathrm{~mm}$.

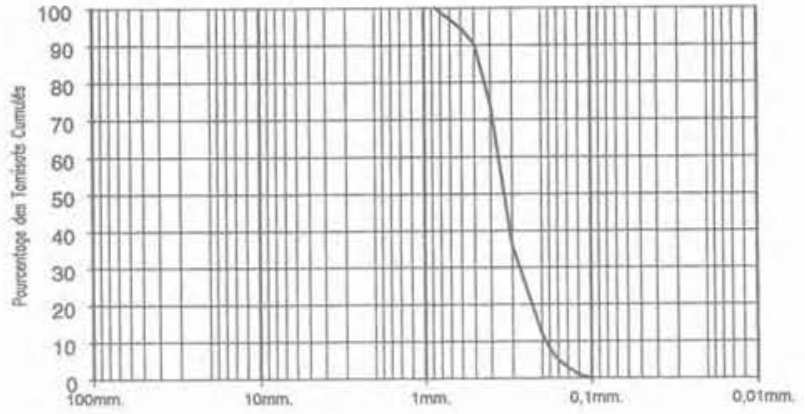

Fig. 1. - Courbe granulométrique du sable de dune. Fig. 1. - Granulometric curve of dune sand.

Le coefficient d'uniformité inférieur à 2, confirme une granulométrie serrée.

$$
C_{u}=\frac{d_{60}}{d_{10}}=1,7
$$

$\mathrm{d}_{60}=$ diamètre de tamis correspondant à $60 \%$ de passants ;

$\mathrm{d}_{10}=$ diamètre de tamis correspondant à $10 \%$ de passants.

Ce sable ne contient pratiquement pas d'éléments fins passant au tamis de $80 \mu \mathrm{m}$. De ce fait, il possède un équivalent de sable au piston, supérieur à 70 .

En résumé ce sable appartient donc à la catégorie $A_{1}$ des sables fins, très propres, dans la classification RTR pour les utilisations routières [12].

Sa finesse $(0,2 / 0,5 \mathrm{~mm})$ et sa granulométrie uniforme lui confèrent des aptitudes médiocres au compactage.

Le liant hydraulique utilisé pour la stabilisation, est constitué de cendres volantes activées par de la chaux : les cendres volantes de lignites proviennent de la centrale thermique d'Arjuzanx dont la composition est présentée dans le tableau 1 [7] ; les caractéristiques de la chaux (chaux de Sauveterre dans le Lot-et-Garonne) satisfont aux directives officielles (passant au tamis de $80 \mu \mathrm{m}$ supérieur à $90 \%$ et teneur en chaux libre supérieure à $50 \%$ ).

Tableau 1. - Analyse chimique des cendres volantes. Table 1. - Chimical analysis of fly-ash.

\begin{tabular}{|c|c|}
\hline Composition chimique & CV Arjuzanx \\
\hline $\mathrm{SiO}_{2}$ & 35 \\
\hline $\mathrm{Al}_{2} \mathrm{O}_{3}$ & 11 \\
\hline $\mathrm{Fe}_{2} \mathrm{O}_{3}+\mathrm{TiO}_{2}$ & 33 \\
\hline $\mathrm{CaO}$ & 10 \\
\hline $\mathrm{MgO}$ & 2 \\
\hline $\mathrm{Na}_{2} \mathrm{O}+\mathrm{K}_{2} \mathrm{O}$ & 1 \\
\hline $\mathrm{SO}_{3}$ & 7 \\
\hline Divers & 1 \\
\hline
\end{tabular}


Les caractéristiques des mélanges sable-liant étudiés correspondent à celles définies par l'optimum Proctor modifié (OPM) [10]. Caractéristiques moyennes OPM des mélanges :

$$
\gamma_{\mathrm{d}}=17,7 \mathrm{kN} / \mathrm{m}^{3} \quad \mathrm{w}_{\text {opm }}=13,5 \%
$$

\section{PROCÉDURE EXPÉRIMENTALE}

\subsection{Préparation des éprouvettes}

Les éprouvettes de sable stabilisé ont été directement carottées dans un moule CBR contenant le matériau compacté à l'OPM. Seule la partie centrale des éprouvettes a été retenue (diamètre : $35 \mathrm{~mm}$, hauteur: $80 \mathrm{~mm}$ ). Celles-ci ont été conservées à température et hygrométrie constantes, $\left(\theta=20^{\circ}\right.$ et $95 \%$ d'hygrométrie).

\subsection{Paramètres étudiés}

Afin de mettre en évidence le rôle joué par les cendres volantes d'une part, et par la chaux d'autre part, nous avons choisi de définir les mélanges par le rapport CV/S. Dans la composition des trois mélanges étudiés, le sable garde une valeur constante $\mathrm{S}=$ cte, la quantité de cendres volantes varie $(\mathrm{CV} / \mathrm{S}=17 \%$, $24 \%$ et $35 \%$ ), ainsi que la composition du liant : $\mathrm{L}_{1}=10 \% \mathrm{CaO}-90 \% \mathrm{CV}$ et $\mathrm{L}_{2}=20 \% \mathrm{CaO}$ - $80 \% \mathrm{CV}$.

L'étude du comportement mécanique de ces mélanges ( 3 pourcentages de $C V / S$ traités par deux compositions de liant) a été suivie en fonction du temps : $0,7,28,90,180$ et 360 jours.

La dispersion inhérente aux différentes étapes de préparation des éprouvettes nous a amené, pour améliorer la précision des résultats, à fabriquer un grand nombre d'éprouvettes (700 environ).

\subsection{Essai triaxial de compression}

Tous les essais ont été effectués, suivant le mode opératoire : essai triaxial [11]. Afin de suivre la variation diamétrale au centre de l'éprouvette durant l'essai, nous avons équipé, après une expérimentation par laser non concluante, une cellule triaxiale classique d'un système optique permettant d'obtenir sur un écran l'image de l'échantillon grossie 20 fois.

Matériel utilisé :

- presse mécanique à vitesse de déplacement constante $(0,5 \mathrm{~mm} / \mathrm{mn})$;

- capteur à induction pour mesure de la force axiale ;

- capteur potentiométrique au $1 / 100 \mathrm{~mm}$ pour la mesure des déformations axiales de l'éprouvette ;

- système optique pour mesure des déformations diamétrales ;

- table traçante pour le tracé des courbes efforts-déformations.

Sur un cylindre d'acier préalablement enrobé d'une couche de sable, nous avons vérifié que l'effet de pénétration de la membrane était insignifiant ou négligeable dans le domaine des contraintes radiales utilisées $(0$ à $0,5 \mathrm{MPa})$.

\section{RÉSULTATS - INTERPRÉTATION}

Après avoir vérifié que, dans le domaine des contraintes étudiées, la courbe intrinsèque est assimilable à une droite, nous avons choisi de représenter les résultats des essais triaxiaux par la droite joignant les sommets des cercles de Mohr de rupture: $p=f(q)$ :

$$
\mathrm{p}=\frac{\sigma_{1}-\sigma_{3}}{2} ; \mathrm{q}=\frac{\sigma_{1}+\sigma_{3}}{2}
$$

Nous allons démontrer que la droite enveloppe des cercles de Mohr $\mathrm{C}_{\mathrm{T}} \mathrm{T}_{1} \mathrm{~T}_{2}$ et la droite joignant les sommets de ces cercles $C_{A} A_{1} A_{2}$ sont concourantes en $C$.

$$
\begin{aligned}
& \frac{\mathrm{C}_{\mathrm{T}} \mathrm{O}_{1}}{\mathrm{C}_{\mathrm{T}} \mathrm{O}_{2}}=\frac{\mathrm{O}_{1} \mathrm{~T}_{1}}{\mathrm{O}_{2} \mathrm{~T}_{2}}=\frac{\mathrm{R}_{1}}{\mathrm{R}_{2}} \| \\
& \frac{\mathrm{C}_{\mathrm{A}} \mathrm{O}_{1}}{\mathrm{C}_{\mathrm{A}} \mathrm{O}_{2}}=\frac{\mathrm{O}_{1} \mathrm{~A}_{1}}{\mathrm{O}_{2} \mathrm{~A}_{2}}=\frac{\mathrm{R}_{1}}{\mathrm{R}_{2}} \|
\end{aligned}
$$

$\mathrm{CT}$ et $\mathrm{CA}$ sont confondus, d'où :

$$
\begin{aligned}
& \sin \Phi=\frac{O_{1} T_{1}}{C O_{1}}=\frac{R_{1}}{C O_{1}} \| \\
& \tan \Psi=\frac{O_{1} A_{1}}{C O_{1}}=\frac{R_{1}}{C O_{1}} \| \\
& \tan \Phi=\frac{c}{x} \| \\
& \| \Rightarrow c=e \frac{\sin \Phi=\tan \Psi}{\tan \Psi}
\end{aligned}
$$

$\tan \Psi=\frac{e}{x} \|$

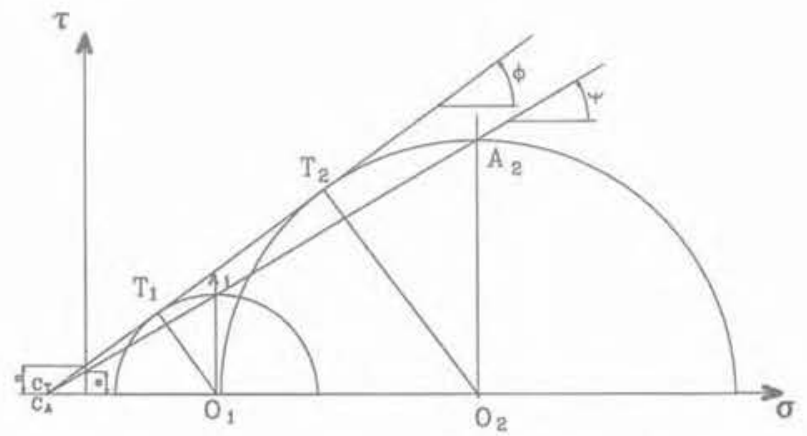

Fig. 2. - Relation entre la droite enveloppe des cercles de Mohr de rupture et la droite joignant les sommets des cercles.

Fig. 2. - Straight line tangent to the Mohr failure circles and straight line connecting the vertex of each cercle. 
Les caractéristiques mécaniques $\Phi$ et c sont telles que :

$$
\Phi=\operatorname{Arc} \sin [\tan \Psi] \quad c=\frac{e}{\sqrt{1-\tan ^{2} \Psi}}
$$

\subsection{Caractéristiques mécaniques du sable pur}

Les résultats des essais triaxiaux effectués sur le sable compacté à l'OPM sont représentés sur la figure 3.

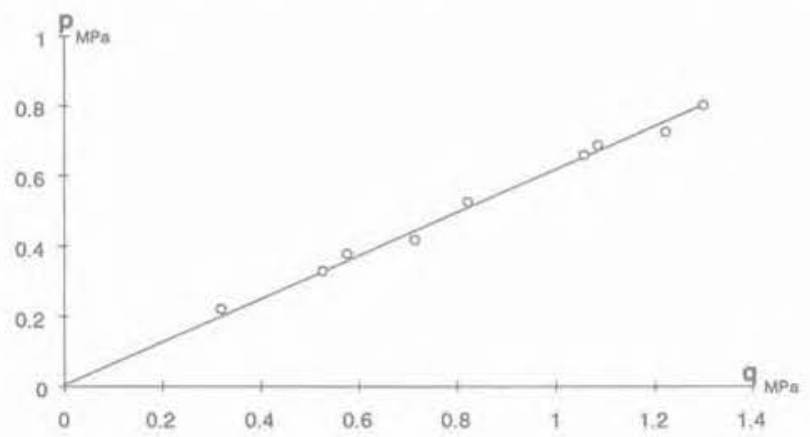

Fig. 3. - Droite $p=f(q)$ du sable pur.

Fig. 3. - Straight line $p=f(a)$ of pure sand.

Sable pur:

$$
\begin{array}{ll}
\Psi=31^{\circ} & e=0,005 \mathrm{MPa} \\
\Phi=38^{\circ} & \mathrm{c}=0,006 \mathrm{MPa}
\end{array}
$$

\subsection{Evolution des caractéristiques mécaniques $\Phi$ et $\mathrm{c}$ aux âges inférieurs à sept jours}

Pour l'ensemble des mélanges (sable + liant) que nous qualifions de frais ( $t<7 \mathrm{j}$ ), les résultats des essais triaxiaux obtenus, sont très proches les uns des autres (fig. 4). Nous notons, pour ces mélanges, une amélioration par rapport aux caractéristiques mécaniques du sable pur (tableau 2).

La faible augmentation de l'angle de frottement interne $\Phi$ peut s'expliquer par le caractère frottant limité des fines ajoutées (CV), celle de la cohésion c par la lenteur du phénomène de prise du liant utilisé. En effet, la chaux solubilise lentement la silice et lalumine contenues dans les cendres volantes pour donner des silicates et aluminates de chaux.

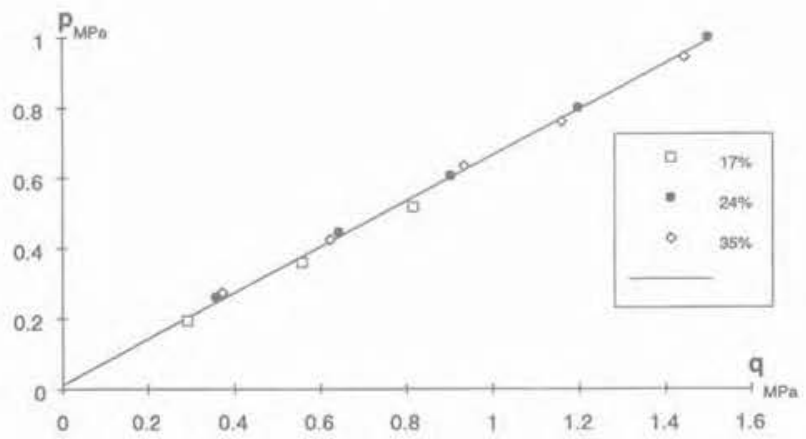

Fig. 4. - Droite $p=f(q)$ des mélanges frais.

Fig. 4. - Straight line $p=f(q)$ of fresh mixtures.
Tableau 2. - Caractéristiques mécaniques du sable pur et des mélanges frais.

Table 2. - Mechanical caracteristics of pure sand and of fresh mixtures.

\begin{tabular}{|c|c|c|}
\hline & $\Phi$ & $\mathrm{C}_{\mathrm{MPa}}$ \\
\hline Sable & $38^{\circ}$ & 0,006 \\
\hline Mélange frais & $41^{\circ}$ & 0,014 \\
\hline
\end{tabular}

4.3. Evolution des caractéristiques mécaniques $\Phi$ et c pour des âges égaux et supérieurs à sept jours

Nous avons constaté que les droites $p=f(q)$ évoluaient, en fonction du temps, suivant un parallélisme, ceci quel que soit la valeur du rapport $\mathrm{CV} / \mathrm{S}$ et la composition du liant comme l'indique les figures 5 , $6,7,8,9$ et 10 .

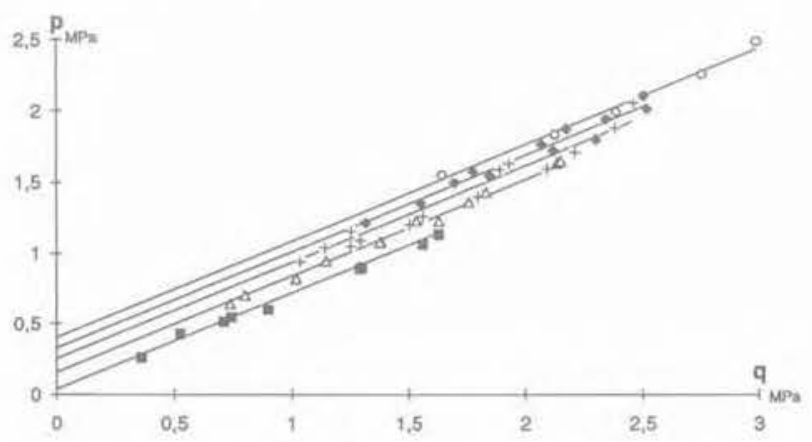

Fig. 5. - Droites $p=f(q)$ Liant $L, C V / S=17 \%$. Fig. $5 .-$ Straight lines $p=f(q)$ hydraulic binder one CV/S $=17 \%$.

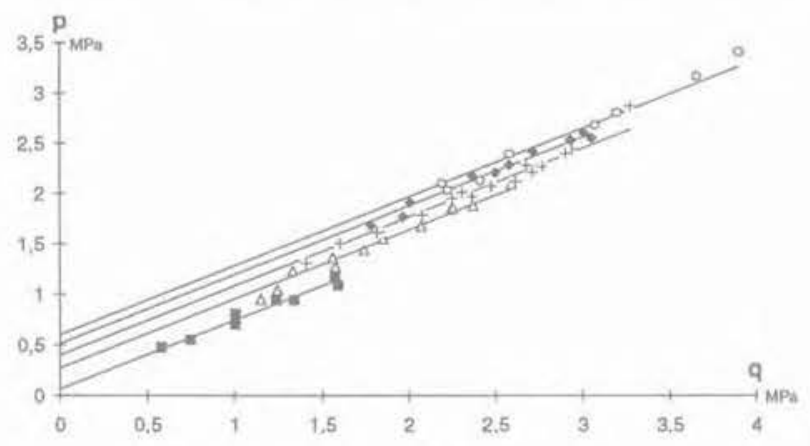

Fig. 6. - Droites $p=f(q)$ Liant $L, V C / S=24 \%$. Fig. 6. - Straight lines $p=f(q)$ hydraulic binder one CV/S $=24 \%$.

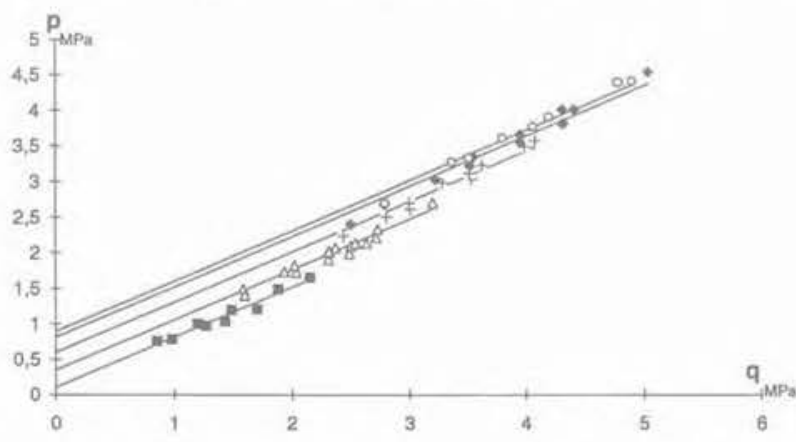

Fig. 7. - Droites $p=f(q)$ Liant $L, C V / S=35 \%$. Fig. 7. - Straight lines $p=f(q)$ hydraulic binder one CV/S $=35 \%$. 


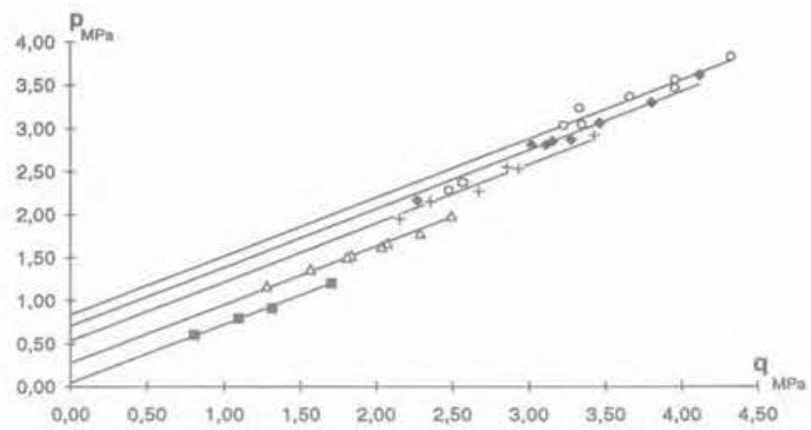

Fig. 8. - Droites $p=f(q)$ Liant $L_{2} V C / S=17 \%$. Fig. 8. - Straight lines $p=f(q)$ hydraulic binder two $C V / S=17 \%$.

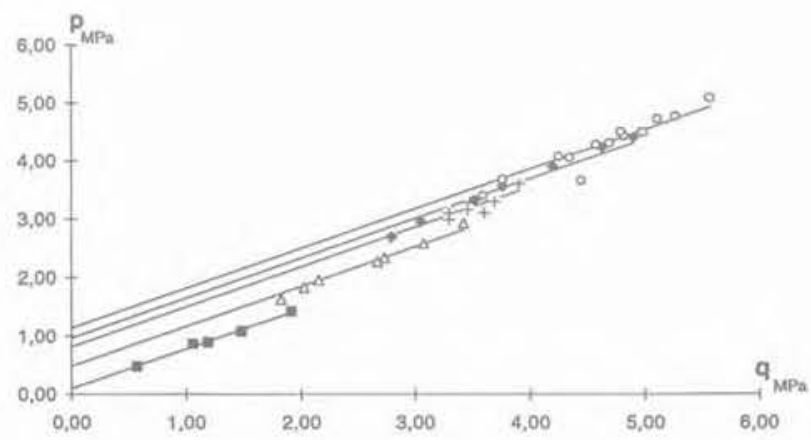

Fig. 9. - Droites $p=f(q)$ Liant $L_{2} V C / S=24 \%$.

Fig. 9. - Straight lines $\rho=f(q)$ hydraulic binder two $C V / S=24 \%$.

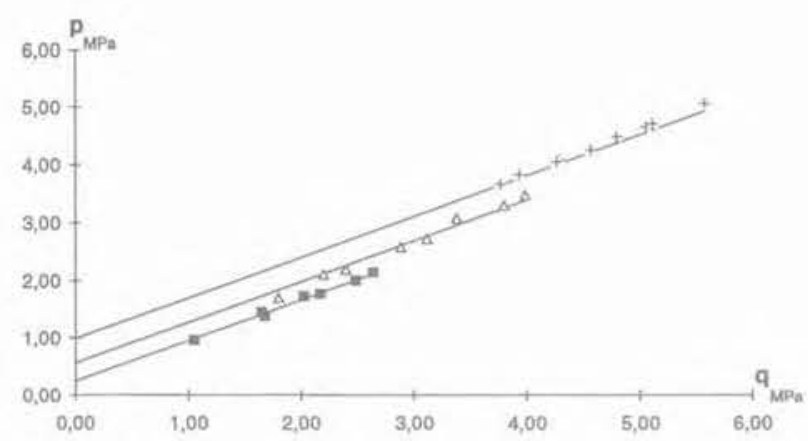

Fig. 10. - Droites $p=f(q)$ Liant $L_{2} V C / S=35 \%$.

Fig. 10. - Straight lines $p=f(q)$. hydraulic binder two $C V / S=35 \%$.

\subsubsection{Angle de frottement interne $\Phi$}

L'évolution de l'angle de frottement interne (dont les valeurs sont regroupées dans le tableau 3) correspond à une amélioration de $5^{\circ}$ par rapport au sable pur, pour les rapports $\mathrm{CV} / \mathrm{S}=17 \%$ et $24 \%$, et une amélioration légèrement supérieure à $7^{\circ}$, pour un remplissage des vides du squelette du sable plus important $(\mathrm{CV} / \mathrm{S}=35 \%)$, qui n'atteint pas toutefois le remplissage maximal théorique.

Nous pouvons donc en déduire que pour les rapports $\mathrm{CV} / \mathrm{S}$ les plus couramment utilisés dans la stabilisation d'un sable, l'angle de frottement interne peut être
Tableau 3. - Variation de l'angle de frottement interne $\Phi$. Table 3. - Variation of internal friction angle $\Phi$.

\begin{tabular}{|c|c|c|c|}
\hline $\begin{array}{c}\text { Liant } \\
\text { CV/S }\end{array}$ & $17 \%$ & $24 \%$ & $35 \%$ \\
\hline $10-90$ & $43^{\circ}$ & $43^{\circ}$ & $45^{\circ}$ \\
\hline $20-80$ & $43^{\circ}$ & $43^{\circ}$ & $45^{\circ}$ \\
\hline
\end{tabular}

considéré comme constant en fonction des trois variables étudiées, car le dosage usuel en cendres volantes, n'atteindra pas $35 \%$.

\subsubsection{Cohésion}

La cohésion, caractéristique essentiellement liée au phénomène de prise et de durcissement du liant est une fonction croissante des trois variables (temps, \% $\mathrm{CV}, \% \mathrm{CaO}$ ).

Les courbes de la variation de la cohésion en fonction du temps $c=f(t)$ (fig. 11 et 12) indiquent que la cohésion qui se manifeste dès sept jours subit une augmentation maximale entre sept et vingt-huit jours. Pourtant à vingt-huit jours, la cohésion, quel que soit le mélange, ne représente que $40 \%$ de la cohésion à trois cent soixante jours. La réaction d'hydraulicité, phénomène de prise et durcissement, se poursuit donc

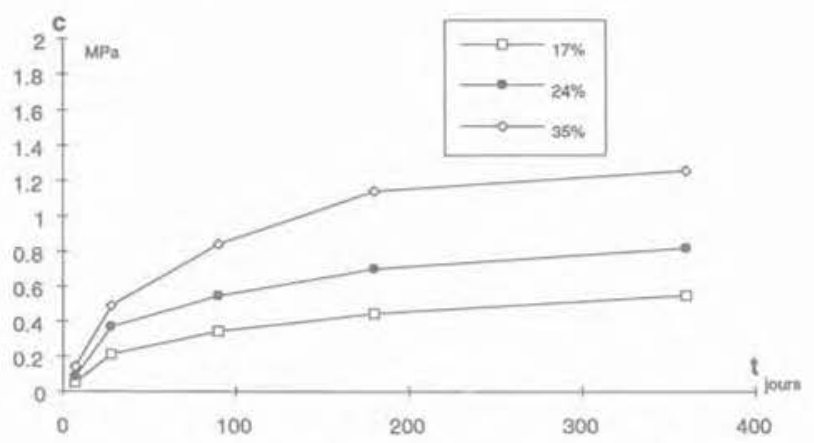

Fig. 11. - Variation de la cohésion en fonction du temps. Liant $L_{1}$.

Fig. 11. - Variation of cohesion versus time. Binder one.

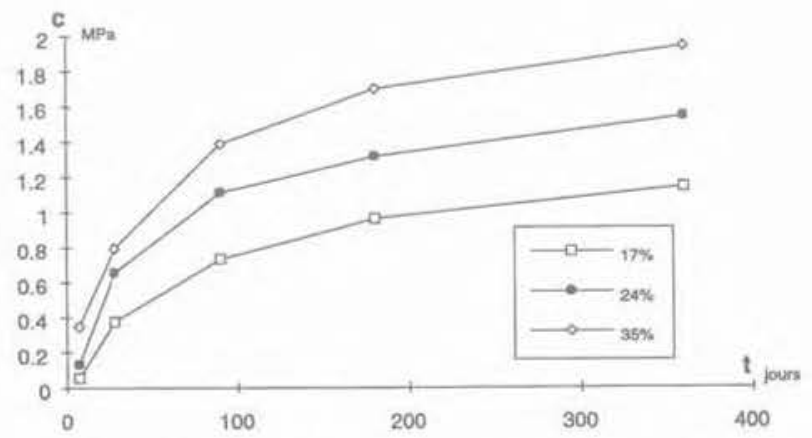

Fig. 12. - Variation de la cohésion en fonction du temps. Liant $\mathrm{L}_{2}$

Fig. 12. - Variation of cohesion versus time. Binder two. 
activement en fonction du temps pour tendre vers une valeur asymptotique vers trois cent soixante jours. A cent quatre-vingt jours, la cohésion atteint 80 à $90 \%$ de la cohésion à un an.

L'expression des résultats de la variation de la cohésion en fonction du temps, sous la forme du rapport $\mathrm{C} / \mathrm{C}_{\propto}$ (cohésion à un âge quelconque sur la cohésion à un an), nous semble plus approprié aux applications pratiques. L'étude du rapport $\mathrm{C} / \mathrm{C}_{\alpha}$, en fontion du logarithme de t (fig. 13 et 14), montre que pour les deux liants, cette fonction présente une certaine linéarité entre sept et trois cent soixante jours. Nous pouvons approximer sans grande erreur, en une seule droite, les trois courbes pour chaque liant.

Ces droites permettraient de déterminer la cohésion à un âge quelconque, connaissant celle à sept jours.

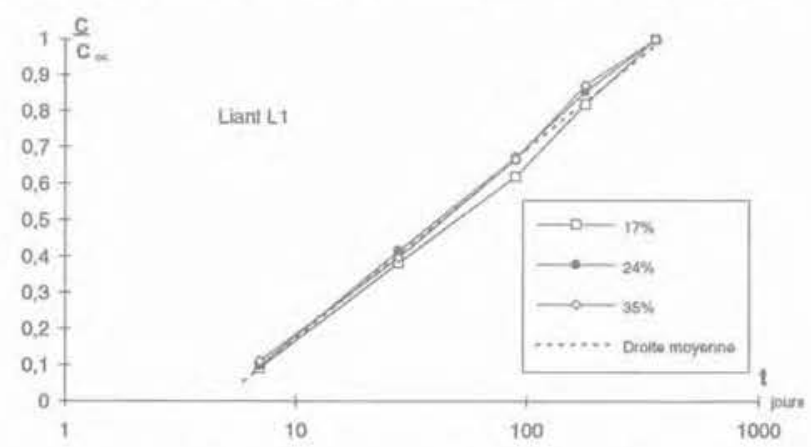

Fig. 13. $-C / C_{\alpha}$ en fonction du temps. Liant $L_{1}$. Fig. 13. $-C / C_{\propto}$ as a function of time. Binder one.

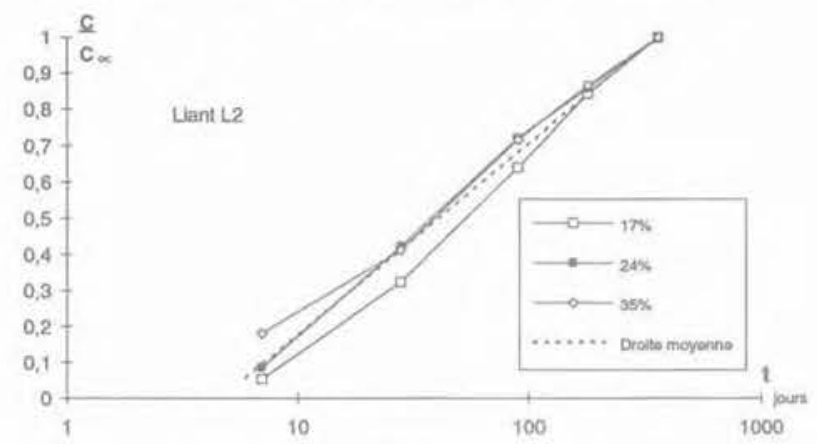

Fig. 14. $-C / C_{\propto}$ en fonction du temps. Liant $L_{2}$. Fig. 14. - C/C $\propto$ as a function of time. Binder two.

La détermination des caractéristiques mécaniques $\Phi$ et c, nécessite un appareillage spécifique, et son interprétation, un minimum de connaissances théoriques. Des lois précédentes concernant l'angle de frottement interne et la cohésion, nous déduisons la loi de variation de la compression simple $\mathrm{R}_{\mathrm{c}}$ et abordons l'étude de la résistance à la traction $\mathrm{R}_{t}$ par le biais du rapport $R_{t} / R_{c}$. Ainsi, nous rendons l'utilisation des résultats théoriques ci-dessus aisément accessibles à un plus grand nombre de praticiens car l'essai de compression simple $R_{c}$ est très facilement réalisable.

\section{UTILISATIONS PRATIQUES DES RÉSULTATS}

5.1. Résistance à la compression simple Compte tenu de la loi de variation de $\Phi$, nous pouvons déduire que $\mathrm{R}_{c}$ suit la même loi de variation que la cohésion $\left(R_{c}=2 c \operatorname{tg}(\pi / 4+\Phi / 2)\right)$ pour les variables : temps, pourcentage de $\mathrm{CV} / \mathrm{S}$, pourcentage de chaux.

Nous avons étudié la variation de $\mathrm{R}_{\mathrm{c}}$ sous la forme du rapport $R_{c} / R_{c \alpha}$, car son exploitation nous semble plus intéressante pour les applications pratiques (fig. 15)

La variation de $R_{c} / R_{c \propto}$ en fonction des trois variables temps, $\mathrm{CV} / \mathrm{S}$ et chaux, est définie dans un repère semi-logarithmique par une loi linéaire d'équation :

$$
\mathrm{R}_{\mathrm{c}} / \mathrm{R}_{\mathrm{c} \alpha}=0,64 \log (\mathrm{T} / 10)
$$

$\mathrm{R}_{\mathrm{c}}$ : résistance à la compression à un âge $\mathrm{t}$ quelconque compris entre sept et trois cent soixante jours ;

$\mathrm{R}_{\mathrm{c} \propto}$ : résistance à la compression à troís cent soixante jours ;

$\mathrm{T}$ : variable exprimée en jour $\mathrm{T}=\mathrm{t}+7$.

Cette droite unique permet, à partir d'un essai simple de compression exécuté dès sept jours sur le sable traité, d'extrapoler la résistance de ce sable en fonction de lâge.

Ex : pour un matériau dont $R_{c 7 j}=0,4 \mathrm{MPa}$, l'équation (1) nous donne $R_{c 7 j} / R_{c \propto}=0,0935$.

Nous en déduisons donc que $\mathrm{R}_{c \propto}=4,3 \mathrm{MPa}$.

La connaissance de $\mathrm{R}_{c \propto}$ permet de déduire, à son tour, la résistance à un âge quelconque : $90 \mathrm{j}, 60 \mathrm{j}$.

Pour $R_{c \propto}=4,3 \mathrm{MPa}$, à 90 jours nous avons $\mathrm{R}_{\mathrm{c} 90} / \mathrm{R}_{\mathrm{c} \alpha}=0,63$ donc $\mathrm{R}_{\mathrm{c} 90 \mathrm{j}}=2,7 \mathrm{MPa}$.

Les valeurs de $R_{c \kappa}$ déduites de la droite $R_{c} / R_{c \alpha}$ le sont avec une erreur relative de l'ordre de $10 \%$ (zone d'incertitude définie par rapport aux points expérimentaux extrêmes).

Nous avons vérifié si la droite $R_{c} / R_{c \alpha}=0,64 \log$ $((t+7) / 10)$ était une caractéristique intrinsèque du sable étudié ou si son champ d'application pouvait s'étendre à l'ensemble des sables homométriques de la même catégorie (catégorie $A_{1}$ de la classification RTR) [10]. A ces fins, nous avons relevé d'une part les valeurs de $R_{c}$ obtenues par d'autres chercheurs [2] pour un sable de même catégorie traité par le même liant et d'autre part nous avons fait quelques essais de contrôle avec un sable de Fontainebleau stabilisé au liant $\mathrm{L}_{1}$.

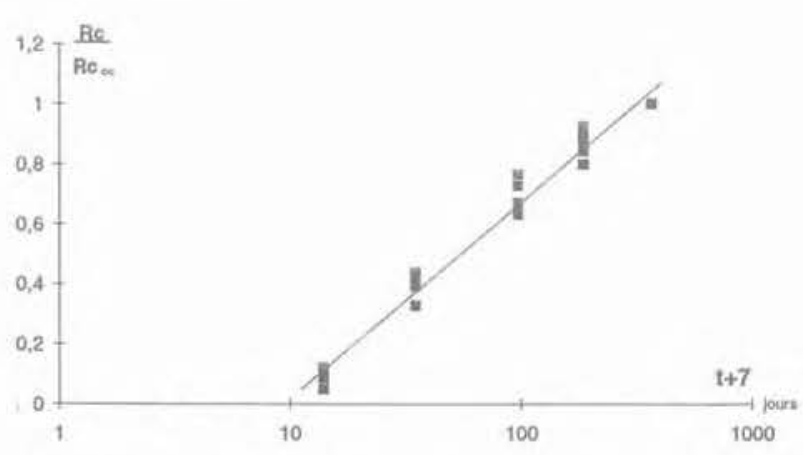

Fig. 15. - Variation de $R_{C} / R_{C \propto}$ en fonction du temps. Fig. 15 - Variation of $R_{C} d R_{C} \propto$ as a function of time. 
Nous avons constaté que l'ensemble de ces points se situe dans l'espace délimité par nos points expérimentaux extrêmes. Ce qui correspond à une marge d'erreur de $10 \%$ par rapport à la valeur moyenne.

Nous pensons donc pouvoir conclure que le domaine d'application de la droite $R_{c} / R_{c \infty}$ ainsi définie peut s'étendre aux sables fins de catégorie $A_{1}$, stabilisé par le liant: cendres volantes-chaux de type $\mathrm{L}_{1}$ ou $\mathrm{L}_{2}$.

Nous retrouvons pour le sable homométrique étudié une loi (1) similaire à celle définie par AVRAM et al. [3] pour des bétons de ciments différents.

\subsection{Etude du rapport $R_{t} / R_{c}$}

Comme $R_{1} / R_{c}=(1-\sin \Phi) /(1+\sin \Phi)$, ce rapport ne dépend que de l'angle de frottement interne $\Phi$, il est donc constant pour les rapports $C V / S$ se situant dans l'intervalle des valeurs les plus utilisées dans la pratique. Pour le sable stabilisé étudié ( $\Phi=$ $43^{\circ}$ pour $\mathrm{CV} / \mathrm{S}=17 \%$ et $24 \%$ ) nous en déduisons que: $R_{t} / R_{c}=1 / 5,3$.

La conclusion $R_{t} / R_{c}=$ cte recoupe celles de diverses publications [2] [5] [6] d'autres chercheurs. Ces derniers, à partir d'essais de compression simple et de traction directe, sur des sables non creux, ou graves traités aux liants hydrauliques, avaient mis en évidence que le rapport $R_{t} / R_{c}$ évoluait dans les limites de $1 / 7$ à $1 / 13$ en fonction de la nature du sable, de la grave et du liant, mais ce rapport restait constant en fonction du temps.

\subsection{Abaque : composition du mélange}

Cet abaque définit le traitement du sable à utiliser pour atteindre les caractéristiques mécaniques désirées connaissant la résistance à la compression simple $\mathrm{R}_{c o}$ du sable pur compacté à l'OPM (fig. 16).

L'étude de la fonction $\mathrm{Y}=\left(\mathrm{R}_{\mathrm{c}} / \mathrm{R}_{\mathrm{co}}\right) /(\mathrm{CV} / \mathrm{S})$ dans un repère semi-logarithmique $(X=\log (t+7))$, se traduit pour les deux liants par deux droites concourantes au point d'abscisse $\mathrm{X}=10$. La pente de ces droites varie avec le type de liant.

En posant $K=R_{c} / R_{c o}$ et $Z=1 / C V / S$ et en donnant différentes valeurs au coefficient $K$, nous obtenons un faisceau de droites $\mathrm{Y}=\mathrm{KZ}$.

En associant la fonction $\mathrm{Y}=\mathrm{KZ}$ à la fonction $\mathrm{Y}=$ $\mathrm{f}(\mathrm{X})$, nous définissons un abaque. Ce dernier permet, connaissant la résistance $\mathrm{R}_{\mathrm{co}}$ du sable pur compacté à l'OPM, de déduire, selon que l'on choisisse le liant $\mathrm{L}_{1}$ ou $\mathrm{L}_{2}$, la valeur du rapport $\mathrm{CV} / \mathrm{S}$ qui donnera par simple lecture la résistance désirée à un âge donné (âge compris entre sept et trois cent soixante jours).

Ex : Quel pourcentage de CV/S et quel liant utiliser pour qu'un sable stabilisé ait une résistance à la compression simple de $4 \mathrm{MPa}$ à quatre-vingt-dix jours?

Si $R_{c o}=0,04 \mathrm{MPa}$, on aura $\mathrm{K}=\mathrm{R}_{\mathrm{c} 90} / \mathrm{R}_{\mathrm{co}}=100$. Sur l'abaque, nous élevons, à partir du point d'abscisse $t+7=97$ jours, une perpendiculaire à l'axe des abscisses. Cette dernière coupe la droite $\mathrm{D}_{1}$ (liant $L_{1}$ ) en un point $A_{1}$ et la droite $D_{2}$ (liant $L_{2}$ ) en un point $A_{2}$. Par ces points $A_{1}$ et $A_{2}$, nous menons 2 horizontales qui coupent la droite $\mathrm{K}=100$ en 2 points $B_{1}$ et $B_{2}$. De ces derniers, nous abaissons les perpendiculaires à Yaxe des abscisses. Les points $\mathrm{C}_{1}$ et $C_{2}$ ainsi définis nous donnent les valeurs du rapport $\mathrm{CV} / \mathrm{S}$ selon le type de liant ;

- pour un liant $L_{1}$, nous aurons besoin d'un rapport $\mathrm{CV} / \mathrm{S}=23,5 \%$;

- pour un liant $\mathrm{L}_{2}$, un rapport de $13 \%$ de $\mathrm{CV} / \mathrm{S}$ suffit pour atteindre $R_{c}=4 \mathrm{MPa}$ à $90 \mathrm{j}$.

Nous pensons, d'après les résultats précédents et les vérifications effectuées, que le champ d'utilisation de cet abaque peut s'étendre à l'ensemble des sables homométriques de catégorie $\mathrm{A}_{1}$.

\section{CONCLUSION}

Le suivi de l'évolution de la courbe intrinsèque d'un sable homométrique stabilisé, par le liant $\mathrm{CV}-\mathrm{CaO}$ en fonction de trois variables : temps, \% $\mathrm{CV}$, \% $\mathrm{CaO}$. nous permet de conclure que langle de frottement interne $\Phi$ du sable stabilisé est essentiellement lié à l'allure de la courbe granulométrique $(\Phi=$ constante pour un sable d'une catégorie) et que la cohésion évolue en fonction du temps avec le pourcentage et la nature du liant.

Des résultats des essais triaxiaux, nous avons déduit la loi de variation de la résistance à la compression simple $\mathrm{R}_{c}$, et celle du rapport $\mathrm{R}_{1} / \mathrm{R}_{\mathrm{c}}$ (résistance à la traction sur résistance à la compression). Nos résultats recoupent ceux d'autres chercheurs qui avaient réalisés des essais de compression simple et de traction directe sur d'autres granulats stabilisés.

Guidés par le souci de contribuer à élargir les applications des sables homométriques plus ou moins délaissés mais très répandus, et pour rendre plus facilement accessibles les résultats de nos travaux, nous présentons :

- une relation qui permet, à partir d'un essai simple de compression à sept jours, d'extrapoler, en fonction de l'áge, la résistance d'un sable fin de catégorie $\mathrm{A}_{1}$ traité par le liant $\mathrm{CV}-\mathrm{CaO}$;

- un abaque qui donne, à partir de l'essai de compression simple du sable pur compacté à POPM, la composition du mélange (sable + chaux + cendres volantes) qui aurait à un âge donné la résistance désirée.

Nous précisons que la méthode proposée pour la détermination de l'abaque doit pouvoir s'étendre à d'autres catégories de sables (A2, B1, gravier...), de liants (ciments, laitier...) ainsi qu'à d'autres types de caractéristiques mécaniques $\left(R_{t}, E\right)$.

Le domaine d'application des sables homométriques stabilisés pourrait s'étendre, en dehors des souscouches de chaussées, à diverses réalisations : stabilisation des talus, mur de soutènement massif, barrage en terre, etc. 


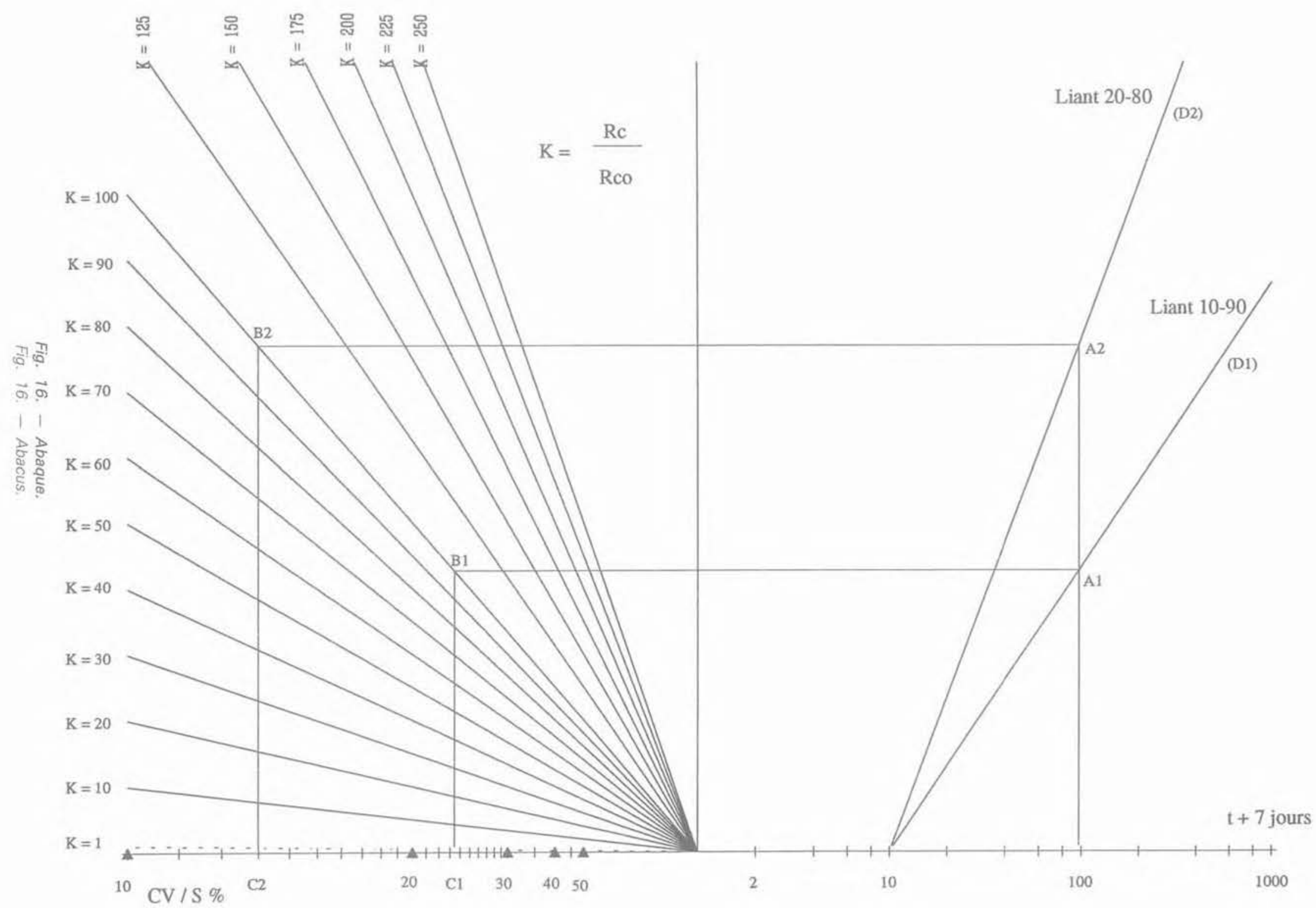




\section{REMERCIEMENTS}

L'auteur remercie M. Y. TCHENG, Directeur du Laboratoire de Construction Civile et Maritime, pour ses conseils et son aide déterminante dans la réalisation de ces travaux, ainsi que M. J.F. COSTE, Directeur du Laboratoire Central des Ponts et Chaussées, pour sa bienveillante disponibilité.

\section{BIBLIOGRAPHIE}

[1] ANDRIEUX P., COLOMBEL J.M. (1976), Utilisation des cendres volantes en technique rou. tière. Bulletin de liaison des laboratoires des Ponts et Chaussées, n 83, p. 77.

[2] ANDRIEUX P., COLOMBEL J.-M. (1976), Application des sables aux cendres volantes. Journées d'information du laboratoire central des Ponts et Chaussées, Bordeaux. Diffusion du laboratoire central des Ponts et Chaussées (LCPC), Paris.

[3] AVRAM C. et al. (1981), Concrete strenght and strains. Developments in Civil Engineering, vol. 3, Editeur Elsevier.

[4] BERTOZZI J. (1989), Comportement mécanique d'un sable homométrique stabilisé. Thèse de Doctorat de l'Université de Poitiers, avril 1989, Université de Poitiers.

[5] JEUFFROY G., SAUTEREY R. (1984), Cours de routes. Dimensionnement des chaussées.
Presses de l'Ecole Nationale des Ponts et Chaussées, Paris.

[6] NGUYEN Da Chi et al. (1974), Assises traitées au liant hydraulique. Résultats généraux (chap. VI). Journées d'information du laboratoire central des Ponts et Chaussées, Nantes. Diffusion LCPC, Paris.

[7] CHARBONNAGES DE FRANCE/EDF, Les cendres volantes des centrales thermiques. Document diffusé par les Charbonnages de France/EDF, Paris.

[8] DIRECTION DES ROUTES (1983), Réalisation des assises de chaussées en graves traitées aux liants hydrauliques. Directive du Ministère de IUUbanisme du Logement et des Transports. Diffusion LCPC, Paris.

[9] DIRECTION DES ROUTES (1985), Réalisation des assises de chaussées en sables traités aux liants hydrauliques. Ministère de PUrbanisme du Logement et des Transports. Diffusion LCPC, Paris.

[10] LABORATOIRE CENTRAL DES PONTS ET CHAUSSÉES, Mode opératoire de l'essai Proctor. Diffusé par les Editions Dunod, Paris.

[11] LABORATOIRE CENTRAL DES PONTS ET CHAUSSÉES, Mode opératoire de l'essai triaxial. Diffusé par les Editions Dunod, Paris.

[12] SETRA, Classification RTR. Document réalisé et diffusé par le SETRA et le LCPC, Paris. 